\title{
Cobalt dependence of the magneto-optical response in magnetoplasmonic nanodisks
}

\author{
J. B. González-Díaz, ${ }^{1, a)}$ B. Sepúlveda, ${ }^{2}$ A. García-Martín, ${ }^{1}$ and G. Armelles ${ }^{1}$ \\ ${ }^{1} I M M$-Instituto de Microelectrónica de Madrid (CNM-CSIC), Isaac Newton 8, PTM, Tres Cantos, \\ E-28760 Madrid, Spain \\ ${ }^{2}$ Research Center on Nanoscience and Nanotechnology-CIN2 (CSIC) and CIBER-BBN, \\ 08196 Bellaterra, Spain
}

(Received 28 June 2010; accepted 12 July 2010; published online 30 July 2010)

\begin{abstract}
In this work, we study the plasmon-mediated enhancement of the magneto-optical (MO) activity in the case of $\mathrm{Au} / \mathrm{Co} / \mathrm{Au}$ nanodisks as a function of the Co content. We show that both the spectral shape and amplitude of the complex polar Kerr rotation are modified by the relative amount of gold (diamagnetic) and cobalt (ferromagnetic). We demonstrate that such shape modification is dictated by the predominance of the absorptive or dispersive part of the MO constant in each material, which is intimately related to the origin of their MO activity. (c) 2010 American Institute of Physics. [doi:10.1063/1.3474617]
\end{abstract}

During the past decade there has been a great interest in plasmonic nanoscale objects, motivated by the ability of surface plasmons to surpass the diffraction limit of conventional optics, such capability to localize the electromagnetic field in subwavelength volumes can open paths to envisage recent nanophotonic devices. ${ }^{1,2}$ With the aim of providing optical activity and functionalities to plasmonic structures, noble metal films, and nanostructures have been recently combined with ferromagnetic materials to take advantage of their strong magneto-optical (MO) activity when an external magnetic field is applied. These magnetoplasmonic structures have been employed to improve the limit of detection in surface plasmon resonance (SPR) sensors ${ }^{3}$ or to modulate the transmittance of propagating surface plasmon polaritons (SPP), ${ }^{4}$ based on the nonreciprocal variation in the SPP wave vector with an external magnetic field. ${ }^{5,6}$

Another recent application has been the enhancement of the MO response in nanostructures made out of noble metals and ferromagnets due to the localization of the electromagnetic field induced by the excitation of a localized SPR (LSPR). ${ }^{7-14}$ In these systems the ferromagnet induces the MO activity and the noble metal allows the excitation of nondamped plasmons, which increase the electromagnetic field intensity inside the ferromagnet and, therefore, the MO response of the system. However, very recently it has been shown that pure noble metal nanoparticles can also exhibit a measurable MO activity at low magnetic fields, without the need of any ferromagnetic material if the LSPR is excited. ${ }^{15}$ This effect was found to be a consequence of an increase in the magnetic Lorentz force induced by the large collective movement of the conduction electrons in the nanostructures when the resonance is excited.

In order to analyze the origin of the MO activity with and without ferromagnetic material and how it is manifested in the amplitude and shape of the MO spectra, we present a comprehensive analysis of the MO effects in plasmonic nanostructures with different amounts of the ferromagnetic material. We will show that the ferromagnetic material, not only reduces the magnetic field required to observe appre-

${ }^{a)}$ Electronic mail: juanb@imm.cnm.csic.es. ciable MO effects, but also induces a drastic change in their spectral dependence, despite of the slight modification of the optical response.

To show the dependence of the MO effects on the amount of ferromagnetic materials we have fabricated arrays of randomly distributed $\mathrm{Au} / \mathrm{Co} / \mathrm{Au}$ nanodisks by hole-mask colloidal lithography. ${ }^{16}$ In these samples we have varied the diameter (60 and $110 \mathrm{~nm}$ ) to change the position of the LSPR and the quantity of $\mathrm{Co}$, keeping constant the total thickness of the disk at $32 \mathrm{~nm}$. The Co layer is sandwiched between two identical Au layers and its thickness is $0 \mathrm{~nm}, 6 \mathrm{~nm}$, and $10 \mathrm{~nm}$, respectively. Figure 1(a) shows a typical scanning electron microscope (SEM) image of a $60 \mathrm{~nm}$ diameter sample. From this image it can be seen that the nanodisks concentration is small enough to neglect possible interactions between the disks, which could lead to a broadening of the plasmonic resonance. Figure 1(b) shows the absorption spectra as a function of the Co quantity in the nanodisks. As it can be seen, the absorption spectra have a peak, which corresponds to the LSPR of the disks. As the amount of Co increases, the peak broadens and slightly blueshifts. In addition, Fig. 1(c) displays the normalized magnetization curves as a function of the magnetic field intensity for various Co amounts. These curves evidence the magnetic character of the samples, since samples containing Co display a saturated curve (dots), typical of a ferromagnetic behavior, whereas samples without Co display a linear dependence of the magnetization (squares) with the magnetic field, as is expected from a diamagnetic material like Au. Also in this figure we present their corresponding polar Kerr rotation and ellipticity spectra [Figs. 1(d)-1(f)], which represent the change in the polarization state of the reflected light induced by the applied magnetic field. The spectra were obtained at normal incidence by applying a magnetic field of $1.6 \mathrm{~T}$. As it can be seen, the Co layer strongly modifies the wavelength dependence of the Kerr rotation and ellipticity spectra even though it only slightly modifies the position and shape of the absorption spectra. In detail, the rotation and ellipticity spectra of the Au nanodisks has a peak and an $S$-like structure, respectively, whereas the rotation and ellipticity spectra of the $\mathrm{Au} /$ $\mathrm{Co} / \mathrm{Au}$ nanodisks display the opposite structure. In addition, 

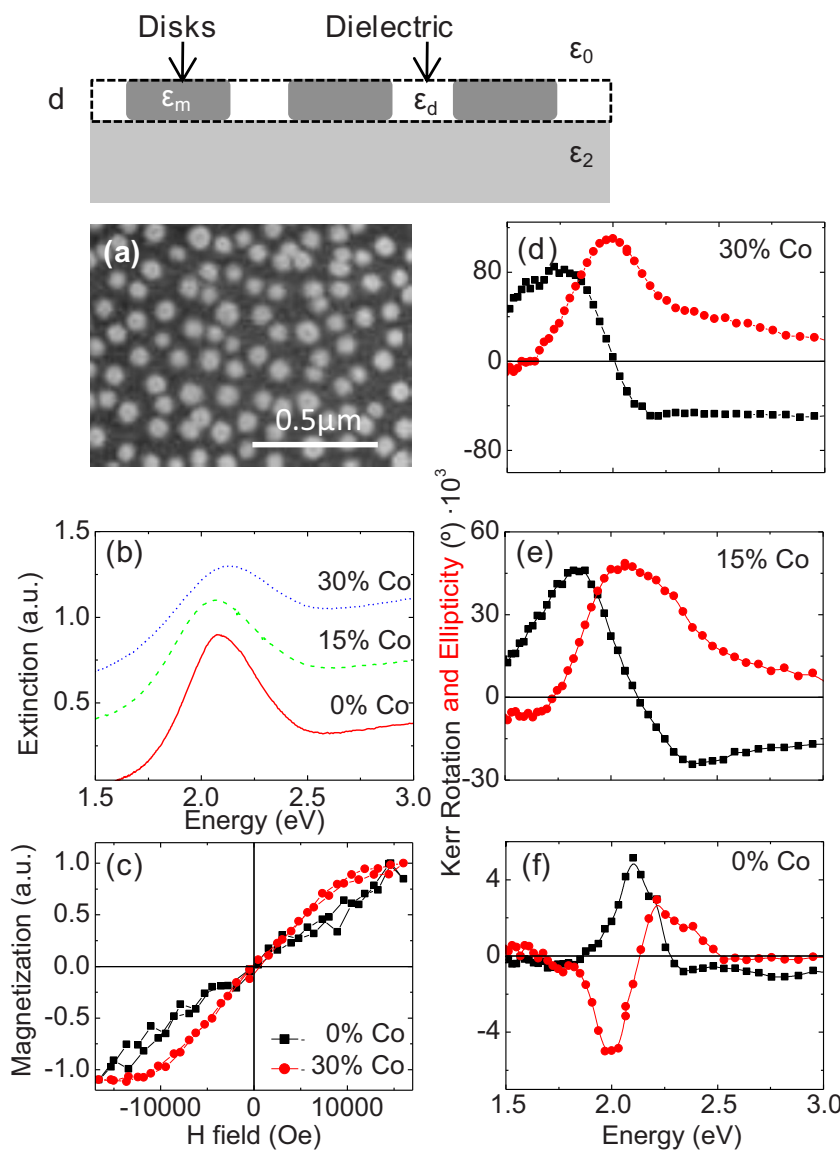

FIG. 1. (Color online) Top: Scheme of the system considered. (a) SEM image of $60 \mathrm{~nm}$ diameter $\mathrm{Au} / \mathrm{Co} / \mathrm{Au}$ nanodisks. (b) Extinction spectra for 60 $\mathrm{nm}$ diameter samples for different percentage of Co within nanodisks. (c) Magnetization as function of magnetic field strength for nanodisks with (red dots) and without (black squares) Co within the nanodisks. [(d)-(f)] Kerr rotation (black squares) and ellipticity (red dots) spectra for samples with different Co amount.

the intensity of the rotation and ellipticity increases with the $\mathrm{Co}$ amount, being in both cases much larger than that of $\mathrm{Au}$ nanodisks. Similar results are obtained in the samples with larger nanodisks diameter, although redshifted spectral structures are observed due to the increase in the nanodisks aspect ratio.

To understand the causes of the spectral modification of the MO activity induced by Co, we consider the different physical mechanisms that give rise to the MO activity in gold and cobalt. Macroscopically, these mechanisms are manifested through the nondiagonal dielectric constants $\left(\varepsilon_{x y}\right)$, therefore correlation between the MO response and the optical and MO dielectric constants of the nanostructure is required. To do so, we consider a simple model consisting of a spherical metal nanoparticle of radius $\alpha$, much smaller than the light wavelength, embedded in a dielectric medium. The complex Kerr rotation can be then expressed in terms of the polarizability tensor $\alpha_{i j}$ of the nanoparticle as follows: ${ }^{15}$

$$
\theta_{K}=\arctan \left(\frac{\alpha_{x y}}{\alpha_{x x}}\right) \approx \frac{3 \varepsilon_{d} \varepsilon_{x y}}{\left(\varepsilon_{x x}-\varepsilon_{d}\right)\left(\varepsilon_{x x}+2 \varepsilon_{d}\right)},
$$

where $\varepsilon_{x x}$ and $\varepsilon_{x y}$ are the optical and MO dielectric constants of the metallic nanoparticle and $\varepsilon_{d}$ the dielectric constant of the external dielectric medium.

For simplicity, we only analyze the ellipticity spectra, since the Kerr rotation can be easily obtained via Kramers-

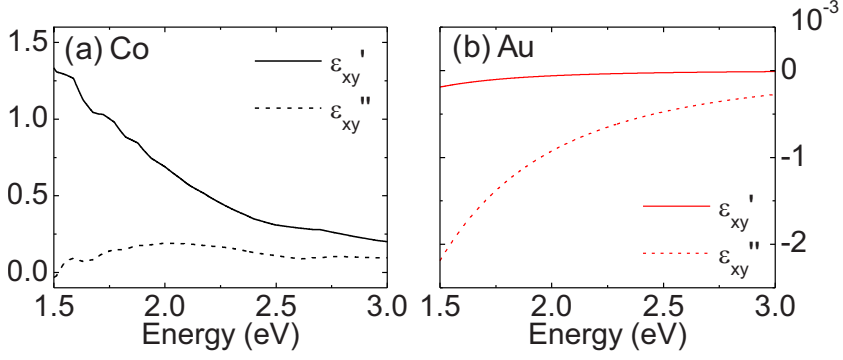

FIG. 2. (Color online) Real and imaginary parts of the MO dielectric constants of (a) cobalt and (b) gold.

Kronig relations. The ellipticity $\varphi_{\mathrm{K}}$ can be expressed in terms of the diagonal $\left(\varepsilon_{x x}=\varepsilon_{x x}^{r e}+i \varepsilon_{x x}^{i m}\right)$ and off-diagonal $\left(\varepsilon_{x y}=\varepsilon_{x y}^{r e}\right.$ $\left.+i \varepsilon_{x y}^{i m}\right)$ elements of the dielectric tensor as follows:

$$
\varphi_{K} \approx \frac{3 \varepsilon_{d}}{\left(\varepsilon_{x x}^{r e}-\varepsilon_{d}\right)^{2}}\left[\frac{\varepsilon_{x y}^{i m}\left(\varepsilon_{x x}^{r e}-\varepsilon_{d}\right)}{\left(\varepsilon_{x x}^{r e}+2 \varepsilon_{d}\right)}-\frac{\varepsilon_{x y}^{r e}\left(2 \varepsilon_{x x}^{r e}+\varepsilon_{d}\right) \varepsilon_{x x}^{i m}}{\left(\varepsilon_{x x}^{r e}+2 \varepsilon_{d}\right)^{2}}\right],
$$

where it has been assumed that the metal has very low absorption losses $\left(\varepsilon_{x x}^{i m} \ll 1\right)$. As a result, from a spectral point of view, if the imaginary part of the MO activity (dispersive part) is larger than the real one (absorptive part) $\left(\varepsilon_{r e}^{x y} \ll \varepsilon_{i m}^{x y}\right)$, the first term in Eq. (2) dominates and the ellipticity shall present an $s$-shape structure (note that this term presents a change in sign in the denominator when approaching $\varepsilon_{r e}^{x x}=-2 \varepsilon_{d}$, which is precisely the condition of LSPR excitations). On the other hand, if the real part of the MO activity is much larger than the imaginary part $\left(\varepsilon_{r e}^{x y} \gg \varepsilon_{i m}^{x y}\right)$, the second term of Eq. (2) dictates the spectral response, and the ellipticity will display a peak structure. In conclusion, the shape differences are originated by the part of the dielectric constant that is modified by the magnetic field.

To better see this, Figs. 2(a) and 2(b) shows the real (continuous line) and imaginary (dashed line) parts of the MO constants of $\mathrm{Co}$ and $\mathrm{Au}$. For Co, these constants were experimentally obtained, whereas those for Au were obtained through a Drude model calculation. It can be observed in this spectral range that the real part of the nondiagonal constant of cobalt is much larger than the imaginary part, being the opposite for gold. This different behavior obeys to the different physical mechanisms giving rise to the MO activity in $\mathrm{Au}$ and Co. For gold, the main contribution to the optical and MO properties comes from conduction electrons, whereas for cobalt the MO activity is mainly due to the spin-orbit interaction of $d$ electrons.

Therefore, for the pure Au nanodisks, $\varepsilon_{r e}^{x y} \ll \varepsilon_{i m}^{x y}$ at the LSPR spectral region and the ellipticity should have an $S$-shape spectral structure, as it is experimentally observed. In contrast, since the MO constants of Co are three orders of magnitude larger than that of $\mathrm{Au}$, the $\mathrm{MO}$ properties of the $\mathrm{Au} / \mathrm{Co} / \mathrm{Au}$ nanodisks are fully determined by the Co part. As a consequence, $\varepsilon_{r e}^{x y} \gg \varepsilon_{i m}^{x y}$ at the LSPR region, which is manifested as a peak structure in the ellipticity.

This simple model gives an accurate explanation of the experimental behavior but in order to confirm these assumptions we calculated the Kerr ellipticity spectra using a scattering matrix algorithm. ${ }^{17}$ The optical and MO constant of Co used in the calculations were obtained from ellipsometry and polar Kerr measurements of continuous films, whereas the MO constant of $\mathrm{Au}$ where simulated using a 

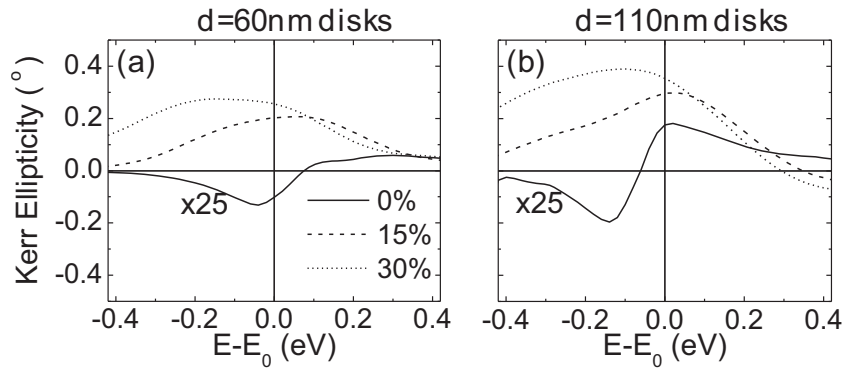

FIG. 3. (a) Theoretical ellipticity curves corresponding to an array of $60 \mathrm{~nm}$ diameter $\mathrm{Au} / \mathrm{Co} / \mathrm{Au}$ nanodisks with several $\mathrm{Co}$ concentrations, from $0 \%$ to $30 \%$ (The spectra for the pure Au nanodisks was multiplied by a factor of 25 in order to become clearly visible). All the spectra are referenced to the spectral position of the LSPR when no magnetic field is applied. (b) Same as in (a) but for $110 \mathrm{~nm}$ diameter nanodisks.

Drude model with the following parameters: $\omega_{p}=3.58 \mathrm{eV}$, $\Gamma=0.065 \mathrm{eV}$, and $\omega_{c}=1.856 \times 10^{-4} \mathrm{eV}$ for a $1.6 \mathrm{~T}$ magnetic field. For the sake of clarity, these simulations, presented in Figs. 3(a) and 3(b), were referenced with respect to their corresponding spectral position of the LSPR $\left(\mathrm{E}_{0}\right)$. They show a spectral dependence in very good agreement with the experimental results, although their intensities are higher than the experimental ones, which is possibly due to a difference in the optical and MO properties of the materials employed in the calculations.

These results highlights the similarities between the MO activity in $\mathrm{Au}$ and $\mathrm{Au} / \mathrm{Co} / \mathrm{Au}$ nanodisks and that observed in MO materials, such as garnets, due to optical transition between atomiclike states of magnetic ions. In these materials part of the MO activity is originated from electric dipole transitions between localized states of magnetic ions and according to its spectral shape can be classified as diamagnetic and paramagnetic transitions. In a diamagnetic transition the MO effects mainly result from the magnetic field splitting of the excited sate and the difference in the absorption coefficient between left and right-handed circular polarized light around that transition has mainly an $S$-shape spectral structure (equivalent to the magnetic field shift in the LSPR wavelength in Au nanodisks). On the other hand, for paramagnetic transitions, the different occupation of the ground state, split by the magnetic field, is the main cause of the MO effects. In this case, the difference in the absorption coefficient between left and right-handed circular polarized light around that transition shows a peak spectral structure (equivalent to the magnetic field modulation of the LSPR absorption in $\mathrm{Au} / \mathrm{Co} / \mathrm{Au}$ nanodisks).
In conclusion, these results confirm the different nature of the $\mathrm{MO}$ activity in $\mathrm{Au}$ and $\mathrm{Au} / \mathrm{Co} / \mathrm{Au}$ nanodisks and how such difference drastically modifies the spectral behavior of the MO effects. While in the former, the MO activity is induced by the collective movement of Au conduction electrons, in the later it is fully dominated by Co and conductions electrons show a negligible contribution. Such contributions entirely explain the different spectral behavior observed when $\mathrm{Co}$ is introduced in the nanodisks.

Financial support from EU Grant No. NMP3-SL-2008214107 (NANOMAGMA), CAM Grant No. S2009/TIC1476 (MICROSERES), and MICINN ("FUNCOAT" CONSOLIDER INGENIO 2010 Grant Nos. CSD2008-00023, MAT2008-06765-C02-01/NAN, and "Ramon y Cajal" program) is gratefully acknowledged.

${ }^{1}$ W. L. Barnes, A. Dereux, and T. W. Ebbesen, Nature (London) 424, 824 (2003).

${ }^{2}$ H. A. Atwater, Sci. Am. 296, 56 (2007).

${ }^{3}$ B. Sepúlveda, A. Calle, L. M. Lechuga, and G. Armelles, Opt. Lett. 31, 1085 (2006)

${ }^{4}$ V. V. Temnov, G. Armelles, U. Woggon, D. Guzatov, A. Cebollada, A. Garcia-Martin, J. M. Garcia-Martin, T. Thomay, A. Leitenstorfer, and R. Bratschitsch, Nat. Photonics 4, 107 (2010).

${ }^{5}$ B. Sepulveda, L. A. Lechuga, and G. Armelles, J. Lightwave Technol. 24, 945 (2006).

${ }^{6}$ J. B. González-Díaz, A. García-Martín, G. Armelles, J. M. García-Martín, C. Clavero, A. Cebollada, R. A. Lukaszew, J. R. Skuza, D. P. Kumah, and R. Clarke, Phys. Rev. B 76, 153402 (2007).

${ }^{7}$ V. I. Safarov, V. A. Kosobukin, C. Hermann, G. Lampel, J. Peretti, and C. Marliere, Phys. Rev. Lett. 73, 3584 (1994).

${ }^{8}$ V. I. Belotelov, L. L. Doskolovich, and A. K. Zvezdin, Phys. Rev. Lett. 98, 077401 (2007).

${ }^{9}$ Y. M. Strelniker and D. J. Bergman, Phys. Rev. B 77, 205113 (2008).

${ }^{10}$ G. A. Wurtz, W. Hendren, R. Pollard, R. Atkinson, L. Le Guyader, A. Kirilyuk, T. Rasing, I. I. Smolyaninov, and A. V. Zayats, New J. Phys. 10, 105012 (2008).

${ }^{11}$ J. B. González-Díaz, A. García-Martín, J. M. García-Martín, A. Cebollada, G. Armelles, B. Sepúlveda, Y. Alaverdyan, and M. Käll, Small 4, 202 (2008).

${ }^{12}$ P. K. Jain, Y. H. Xiao, R. Walsworth, and A. E. Cohen, Nano Lett. 9, 1644 (2009).

${ }^{13}$ C. S. Levin, C. Hofmann, T. A. Ali, A. T. Kelly, E. Morosan, P. Nordlander, K. H. Whitmire, and N. J. Halas, ACS Nano 3, 1379 (2009).

${ }^{14}$ L. Balcells, B. Martinez, O. Iglesias, J. M. García-Martín, A. Cebollada, A. García-Martín, G. Armelles, B. Sepúlveda, and Y. Alaverdyan, Appl. Phys. Lett. 94, 062502 (2009).

${ }^{15}$ B. Sepúlveda, J. B. González-Díaz, A. García-Martín, L. M. Lechuga, and G. Armelles, Phys. Rev. Lett. 104, 147401 (2010).

${ }^{16}$ H. Fredriksson, Y. Alaverdyan, A. Dmitriev, C. Langhammer, D. S. Sutherland, M. Zäch, and B. Kasemo, Adv. Mater. (Weinheim, Ger.) 19, 4297 (2007).

${ }^{17}$ A. García-Martín, G. Armelles, and S. Pereira, Phys. Rev. B 71, 205116 (2005) 